Research Paper

\title{
Effect of Previous Interferon-based Therapy on Recurrence after Curative Treatment of Hepatitis C Virus-related Hepatocellular Carcinoma
}

Tomoko Saito ${ }^{1}$, Tetsuhiro Chiba ${ }^{\circledR}$, Eiichiro Suzuki ${ }^{1}$, Masami Shinozaki², Nobuaki Goto², Naoya Kanogawa1 ${ }^{1}$, Tenyu Motoyama1 ${ }^{1}$, Sadahisa Ogasawara ${ }^{1}$, Yoshihiko Ooka1 ${ }^{1}$ Akinobu Tawada1 ${ }^{1}$ Tatsuo Kanda1, Masaru Miyazaki³, Osamu Yokosuka1

1. Department of Gastroenterology and Nephrology, Graduate School of Medicine, Chiba University, Chiba, Japan;

2. Department of Medicine, Numazu City Hospital, Numazu, Shizuoka, Japan;

3. Department of General Surgery, Graduate School of Medicine, Chiba University, Chiba, Japan.

$\square$ Corresponding author: Tetsuhiro Chiba, M.D., Ph.D. Department of Gastroenterology and Nephrology, Graduate School of Medicine, Chiba University, 1-8-1 Inohana, Chuo-ku, Chiba 260-8670, Japan. Telephone: +81-43-2262083 Fax: +81-43-2262088 E-mail: techiba@faculty.chiba-u.jp.

( ) Ivyspring International Publisher. This is an open-access article distributed under the terms of the Creative Commons License (http://creativecommons.org/ licenses/by-nc-nd/3.0/). Reproduction is permitted for personal, noncommercial use, provided that the article is in whole, unmodified, and properly cited.

Received: 2014.02.05; Accepted: 2014.04.21; Published: 2014.05.07

\begin{abstract}
Previous reports have shown that interferon (IFN)-based therapy decreases the risk of development of hepatocellular carcinoma (HCC) in patients with chronic hepatitis $\mathrm{C}$ virus (HCV) infection. However, it remains to be fully elucidated whether elimination of HCV by IFN-based therapy inhibits HCC recurrence after curative treatment, such as surgical resection and local ablation therapies. In this study, we aimed to clarify the influence of a sustained virological response (SVR) after IFN-based therapy on recurrence and survival after curative treatment of HCC. Fifty-one patients who underwent curative treatment of HCV-related HCC after receiving IFN-based therapy were analyzed retrospectively. They were classified into SVR $(N=14)$ and non-SVR groups $(N=37)$. In the SVR group, serum levels of aspartate aminotransferase and alanine aminotransferase, the indocyanine green retention rate at $15 \mathrm{~min}$, and the percentages of patients with liver cirrhosis and HCV serotype I were significantly lower, whereas serum albumin level and platelet count were significantly higher upon HCC occurrence. Recurrence-free survival (RFS) for the first recurrence was significantly higher in the SVR group $(P<0.01)$. Multivariate analysis showed that SVR at initial HCC treatment $(P<0.01)$ and multiple tumors $(P<0.01)$ are prognostic factors for RFS. Moreover, RFS for the second recurrence showed a similar trend to that for the first recurrence. In conclusion, patients who underwent IFN-based therapy before initial curative treatment of HCC had a favorable clinical outcome compared with non-SVR patients.
\end{abstract}

Key words: HCC; HCV; IFN; recurrence; SVR.

\section{Introduction}

Hepatocellular carcinoma (HCC) is one of the most fatal cancers worldwide [1]. HCC develops frequently in patients with cirrhosis caused by hepatitis $B$ virus infection, hepatitis $\mathrm{C}$ virus (HCV) infection, and alcohol abuse [2]. Because chronic HCV infection is a major cause of HCC, HCV eradication is considered to be quite important for preventing $\mathrm{HCC}[3,4]$.
Interferon (IFN) has been utilized as an antiviral drug for HCV eradication for approximately three decades. It has been documented that patients with a sustained virological response (SVR) during IFN-based therapy, including combination therapy with peg-IFN and ribavirin, show not only histological improvement through HCV eradication but also a decrease in the 
risk of HCC development [5-7]. On the other hand, patients who show SVR after termination of IFN-based therapy often develop HCC several years after HCV eradication [8, 9]. Recently, it was stated that IFN-based therapy after curative treatment could impact HCC development and HCC recurrence. However, only a few studies have investigated whether previous IFN-based therapy is helpful for preventing HCC recurrence after curative treatment of HCC $[10,11]$.

We investigated clinical outcomes after curative treatment for HCC in patients who had received IFN-based therapy for HCV eradication before initial HCC occurrence. We also examined the prognostic factors for HCC recurrence in these patients.

\section{Methods}

\section{Patients}

We assessed 79 patients (51 patients in Chiba University Hospital and 28 patients in Numazu City Hospital) diagnosed with HCV-related HCC between 2003 and 2012 with a history of IFN-based therapy before curative treatment of HCC, which included such as radiofrequency ablation (RFA), percutaneous ethanol injection (PEI), and hepatic resection. Twenty-eight patients (4 SVR patients and 24 non-SVR patients), including 14 with local recurrence, 3 with recurrence within 6 months after treatment, and 11 with not undergone suitable follow-up, were excluded (Fig. 1). This study was conducted in accordance with the Helsinki Declaration and approved by the Ethics Committee of Graduate School of Medicine, Chiba University and Numazu City Hospital. Informed consent was obtained from each patient.

\section{Definition of SVR}

SVR was defined as undetectable serum HCV RNA 24 weeks after the end of treatment [12].

\section{Diagnosis of liver cirrhosis and HCC}

The diagnosis of cirrhosis was made on the basis of the following criteria: (i) histological examination of biopsy specimens; (ii) clinical manifestation, including gastroesophageal varices, encephalopathy, and ascites; and (iii) thrombocytopenia accompanied by findings suggestive of cirrhosis, such as splenomegaly [13]. HCC was diagnosed on the basis of histopathological examination or the practice guidelines set by the American Association for the Study of Liver Diseases (AASLD) [14, 15].

\section{Treatment and detection of recurrence}

There was no significant difference in the period from IFN treatment to initial HCC development in SVR patients and non-SVR patients (67.3 \pm 47.0 month's vs $75.1 \pm 54.2$ months). Treatment of initial HCC was conducted according to the size and number of HCC tumors and hepatic functional reserve assessed by the Child-Pugh classification. If local ablation was chosen, treatment was repeated until complete necrosis of all HCC lesions with a margin of $>5$ mm was confirmed by dynamic computed tomography (CT). Patients were followed up at our outpatient clinic every 3-6 months by monitoring biochemical and hematological data. Patients were screened using dynamic CT and ultrasonography. If HCC recurrence was suspected, further examinations using magnetic resonance imaging, angiography, and tumor biopsy were performed to confirm the diagnosis. Cases with local recurrences (defined as recurrence at the lesion adjacent to the original tumor) were excluded from the analyses.

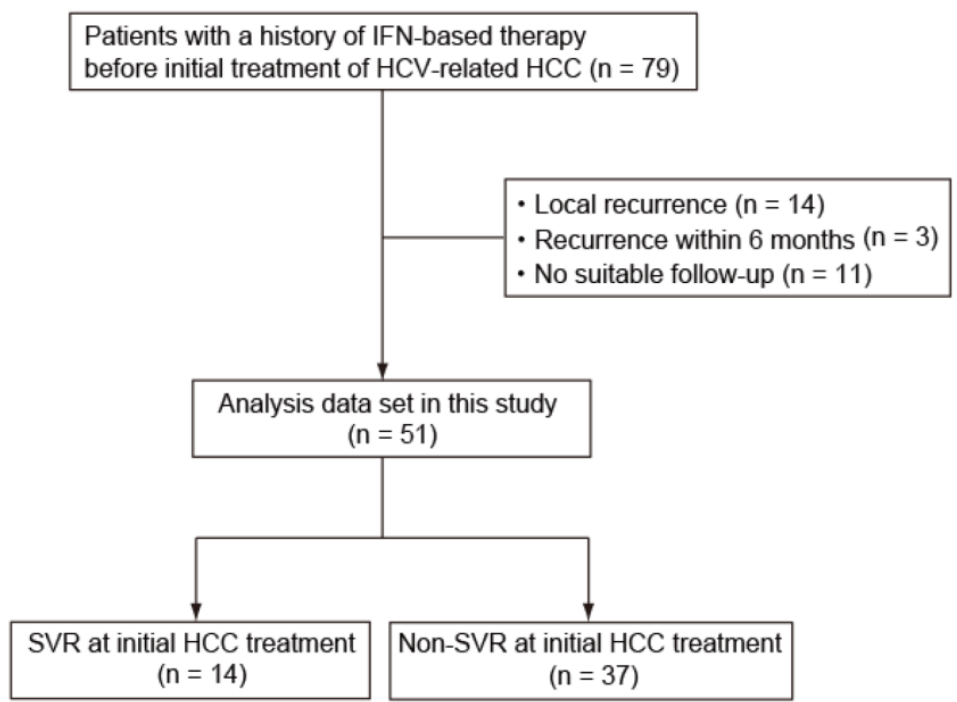

Figure I. Patients enrolled in this study. HCV, hepatitis C virus; IFN, interferon; HCC, hepatocellular carcinoma; SVR, sustained virological response. 


\section{Statistical analysis}

Statistical analyses were performed using SPSS version 19.0 (SPSS Inc. Chicago, IL, USA). Data are the mean \pm SD. Statistical differences between two groups were evaluated by the Student's t-test and chi-square test. If the variances were found not to be equal between the two groups, the result of t-tests was adjusted by Welch's correction. Recurrence-free survival (RFS) was calculated using the Kaplan-Meier method. The prognostic relevance of clinical variables was evaluated by univariate analysis with the log-rank test and by multivariate Cox regression. Variables associated with RFS in univariate analysis with $P$ values of $<0.10$ were analyzed further for multivariate analysis. $P$ values of $<0.05$ were considered significant.

\section{Results}

\section{Patient characteristics}

Of 79 patients with a history of IFN-based therapy before initial curative treatment of HCV-related HCC, 51 patients were considered eligible for the analysis. The number of patients treated with RFA, PEI, and resection was 37, 7, and 7, respectively. They were divided into two groups; SVR $(n=14)$ and non-SVR ( $\mathrm{n}=37$ ) (Fig. 1). Clinical variables compared between SVR patients and non-SVR patients are shown in Table 1. Serum levels of aspartate aminotransferase (AST) and alanine aminotransferase (ALT), the indocyanine green retention rate at $15 \mathrm{~min}$ (ICG $R_{15}$ ), and the percentages of patients with liver cirrhosis and HCV serotype 1 were significantly lower in SVR patients than in non-SVR patients. In contrast, the serum albumin level as well as platelet count were significantly higher in SVR patients than in non-SVR patients.

\section{Prognosis after treatments}

RFS after curative treatment was evaluated. RFS was significantly different between the two groups $(P$ $<0.01$ ) (Fig. 2A). During a median follow-up of 46.6 months in the SVR group and 39.5 months in the non-SVR group, 7 of the 14 SVR patients and 32 of the 37 non-SVR patients had HCC recurrence after curative treatment for initial HCC. The 1-, 3-, and 5-year RFS in the SVR group were $100 \%, 82.0 \%$, and $18.8 \%$, respectively; the values in the non-SVR group were $91.9 \%, 24.7 \%, 5.3 \%$, respectively. In contrast, overall survival after curative treatment of HCC was not significantly different between the SVR and non-SVR groups $(P=0.35)$. In accordance with these results, the first recurrence rate of 37 patients treated with RFA was lower in the SVR group $(n=10)$ than in the non-SVR group $(\mathrm{n}=27)(P<0.01)$ (Fig. 2B). To elucidate the prognostic relevance of clinical variables for recurrence, we performed univariate and multivariate analyses (Table 2). Multivariate analysis revealed that SVR at initial HCC treatment $(P<0.01)$ and multiple tumors $(P<0.01)$ showed a significant correlation with the first recurrence (Table 2).

Next, we investigated the effect of previous IFN-based therapy on the second recurrence of HCC. Of 39 patients with first recurrence, 26 received curative treatment such as local ablation therapy and hepatic resection. After excluding the patients showing a second recurrence within 6 months after the first recurrence or local recurrence $(n=11), 15$ patients $(2$ SVR patients and 13 non-SVR patients) were assessed further. Non-SVR patients showed recurrence after a median interval of 12.5 (range, 6.9-28.8) months after curative treatment for the first recurrence, but the 2 SVR patients showed recurrence after 36.0 months and 48.1 months.

A

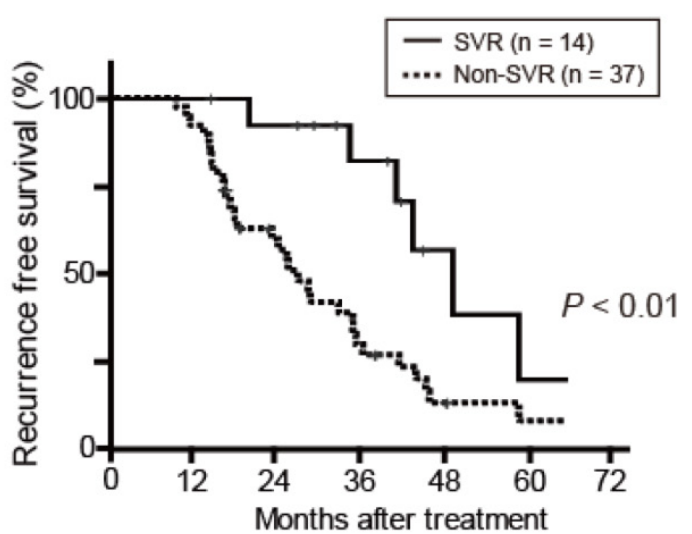

B

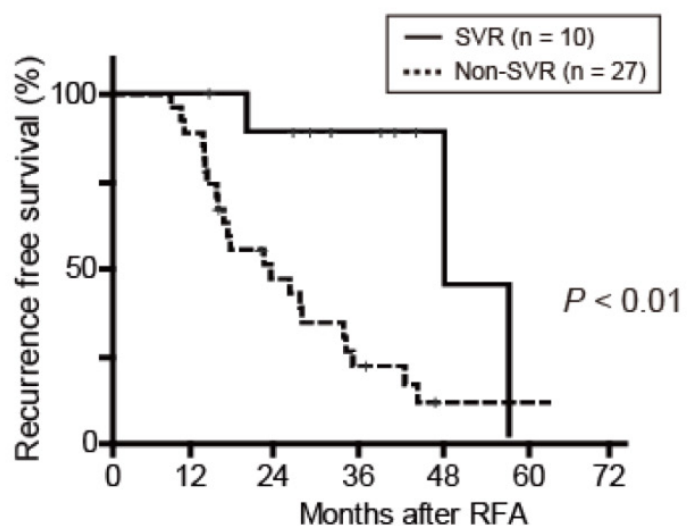

Figure 2. (A) Recurrence-free survival (RFS) after curative treatment for $\mathrm{HCC}$ in $5 \mathrm{I}$ patients. (B) RFS after curative RFA treatment for $\mathrm{HCC}$ in 37 patients. RFA, radiofrequency ablation; SVR, sustained virological response. 
Table I. Baseline patient characteristics at initial HCC treatment.

\begin{tabular}{llll}
\hline Clinical feature & SVR group $(\mathrm{n}=14)$ & non-SVR group $(\mathrm{n}=37)$ & $P$-value \\
\hline Age (median, years) & $72(57-77)$ & $67(50-80)$ & 0.07 \\
Sex (male/female) & $13 / 1$ & $25 / 12$ & 0.06 \\
AST (median, IU/L) & $24(15-44)$ & $74(23-193)$ & $<0.01^{*}$ \\
ALT (median, IU/L) & $18(10-44)$ & $64(27-190)$ & $<0.01^{*}$ \\
Total bilirubin (median, mg/dL) & $0.9(0.6-1.6)$ & $1.0(0.3-2.1)$ & 0.59 \\
Albumin (median, g/dL) & $4.25(2.9-4.5)$ & $3.7(3.0-4.6)$ & $<0.01^{*}$ \\
Platelet count (median, x10 $/ \mathrm{uL})$ & $15.9(9.3-23.4)$ & $10.8(4.0-28.5)$ & $0.02^{*}$ \\
Prothrombin time (median, \%) & $92.1(57.1-116.7)$ & $87.0(55.0-118.0)$ & 0.62 \\
ICG $\mathrm{R}_{15}$ (median, \%) & $12.2(2.9-27.0)$ & $18.0(5.0-58.3)$ & $0.01^{*}$ \\
Progression to cirrhosis (CH/LC) & $12 / 2$ & $19 / 18$ & $0.02^{*}$ \\
HCV serotype (1/2/unknown) & $4 / 5 / 5$ & $25 / 7 / 5$ & $0.02^{*}$ \\
Maximal tumor size (median, cm) & $1.9(1.0-5.0)$ & $2.0(0.8-4.8)$ & 0.55 \\
Tumor number (solitary/multiple) & $9 / 5$ & $26 / 11$ & 0.46 \\
AFP (median, ng/mL) & $5.0(2.0-26170)$ & $25.1(1.0-11567.0)$ & 0.19 \\
DCP (median, mAU/mL) & $21.0(110.0-143.0)$ & $25.0(7.0-531.0)$ & 0.21 \\
\hline
\end{tabular}

"Statistically significant. AST, aspartate aminotransferase; ALT, alanine aminotransferase; ICG, Indocyanine green; $\mathrm{CH}$, chronic hepatitis; LC, liver cirrhosis; HCV, hepatitis C virus; AFP, alpha-fetoprotein; DCP, des-gamma-carboxy prothrombin.

Table 2. Risk factors for the first recurrence after curative HCC treatment.

\begin{tabular}{lllll}
\hline & Univariate analysis & & Multivariate analysis \\
& Hazard ratio $(95 \% \mathrm{CI})$ & $P$-value & Hazard ratio $(95 \% \mathrm{CI})$ & $P$-value \\
\hline Age $(>65$ years $)$ & $0.76(0.41-1.46)$ & 0.76 & & $<0.01^{*}$ \\
Sex (male) & $0.71(0.35-1.45)$ & 0.35 & $0.21(0.08-0.52)$ & 0.88 \\
SVR at initial HCC & $0.29(0.12-0.70)$ & $<0.01^{*}$ & $0.83(0.09-7.53)$ & 0.36 \\
AST $(>50$ IU/L) & $2.81(1.40-5.64)$ & $<0.01^{*}$ & $1.48(0.63-3.48)$ & 0.24 \\
ALT $(>50 \mathrm{IU} / \mathrm{L})$ & $2.68(1.36-5.30)$ & $<0.01^{*}$ & & \\
Total bilirubin $(>1.0 \mathrm{mg} / \mathrm{dL})$ & $0.71(0.37-1.37)$ & 0.30 & $0.60(0.26-1.40)$ & \\
Platelet count $\left(<10 \mathrm{x} 10^{4} / \mathrm{uL}\right)$ & $0.80(0.41-1.58)$ & 0.52 & & $<0.01^{*}$ \\
Albumin $(<3.5 \mathrm{~g} / \mathrm{dL})$ & $2.02(0.94-4.31)$ & 0.07 & & \\
Prothrombin time $(<70 \%)$ & $0.90(0.38-2.11)$ & 0.81 & & \\
ICG $\mathrm{R}_{15}(>20 \%)$ & $1.42(0.77-2.64)$ & 0.27 & & \\
Liver cirrhosis & $1.36(0.71-2.61)$ & 0.36 & & \\
Tumor number $(\mathrm{multiple})$ & $2.29(1.15-4.52)$ & $0.02^{*}$ & & \\
Maximal tumor size $(>20 \mathrm{~mm})$ & $0.88(0.46-1.70)$ & 0.70 & & \\
AFP $(>100 \mathrm{ng} / \mathrm{mL})$ & $0.62(0.27-1.42)$ & 0.26 & 0.99 & \\
DCP $(>400 \mathrm{mAU} / \mathrm{mL})$ & $1.00(0.49-2.06)$ & $0.7 .32)$ & & \\
\hline
\end{tabular}

"Statistically significant. SVR, sustained virological response; HCC, hepatocellular carcinoma; ALT, alanine aminotransferase; ICG $\mathrm{R}_{15}$, indocyanine green retention rate at 15 min; AFP, alpha-fetoprotein; DCP, des-gamma-carboxy prothrombin.

\section{Discussion}

IFN-a has been used for the treatment for chronic HCV infection approximately 30 years [16]. The antiviral activity of IFN has been attributed to its ability to inhibit the expression of genes involved in viral replication [17]. IFN-a can also enhance the adaptive and innate immune responses of the host.
On the other hand, IFN-a suppresses HCC development through the cessation of hepatic inflammation and regression of hepatic fibrosis. In addition, IFN-a enhances tumor necrosis factor (TNF)-induced apoptosis of tumor cells [18] as well as activation of TNF-related apoptosis-inducing ligand through promyelocytic leukemia protein induction [19] or death receptor 5 up-regulation and NF-KB inactiva- 
tion [20]. We retrospectively analyzed HCV-related HCC patients with a history of previous IFN-based therapy. We aimed to elucidate the advantage of previous IFN-based therapy after curative treatment of HCC.

At first, we examined differences in characteristics between SVR patients and non-SVR patients. The SVR group had significantly lower levels of AST and ALT than non-SVR patients. This appears to be attributable to decreased liver inflammation after IFN-based therapy. The serum albumin level, platelet count, and ICG $R_{15}$ were significantly higher in the SVR group than in the non-SVR group. The percentages of liver cirrhosis patients at initial treatment of HCC in the SVR and non-SVR groups were $14.2 \%$ and $48.6 \%(P=0.02)$, respectively.

We then analyzed the first recurrence of HCC in SVR patients and non-SVR patients. SVR patients at the time of initial HCC treatment showed a significantly lower prevalence of recurrence than non-SVR patients $(P<0.01)$ (Fig. 2). Previous studies have demonstrated 3-year RFS to be $35.7 \%-56.7 \%$ [21] and $49.6 \%-61.1 \%$ [22]. Considering that 3-year RFS in the SVR group was $>80 \%$ in the present study, SVR before initial HCC treatment could contribute to the suppression of recurrence. SVR patients showed a more favorable prognosis than those without SVR in a study cohort of $69 \mathrm{HCV}$-related HCC patients undergoing hepatic resection [23]. Although RFA was performed in 37 of the $51(72.5 \%)$ patients assessed in the present study, SVR patients among these 37 patients showed a lower recurrence rates than non-SVR patients $(P<0.01)$. With regard to the 7 SVR patients with recurrence after curative treatment, all showed normal values of AST and ALT, platelet count, serum albumin, and prothrombin time at the first recurrence. The median period to the first recurrence among these 7 SVR patients was 47.3 months, but the longest period was as long as 130.0 months. These results raise the possibility that some causes other than hepatic inflammation or fibrosis could induce HCC recurrence even after HCV is eradicated [24]. Taken together, SVR obtained during previous IFN-based therapy before the initial occurrence of HCC contributes to a favorable prognosis regardless of the treatment approach against HCC.

Although Miyatake et al. reported that overall survival rate at 5 years was significantly higher in SVR patients than in non-SVR patients [25], there was no significant difference between 2 groups in this study. This might be due to the fact that recent advances in diagnostic tools and treatment modalities have contributed to the improvement of survival in HCC patients.
Next, we examined the second recurrence of HCC in SVR patients and non-SVR patients. In 15 eligible patients treated satisfactorily against first-recurrence lesions, 2 out of the 2 SVR patients and 10 of the 13 non-SVR patients showed a second recurrence. Non-SVR patients showed recurrence after 6.9-28.8 months, but SVR patients showed recurrence after 36.0 months and 48.1 months. Although the sample size was small, SVR patients had a tendency to show recurrence after a longer interval from the first recurrence than non-SVR patients. Previous studies have reported similar findings regarding the inhibitory effect of IFN on not only the first recurrence but also the second recurrence in patients who underwent resection [25, 26]. Further analyses are necessary to clarify this issue.

Recently, it has been reported that combination therapy with direct-acting antiviral agents (DAAs), such as a nonstructural protein (NS) 3 inhibitor and a NS5A inhibitor without peg-IFN and ribavirin, was well tolerated and achieved a high prevalence of SVR $[27,28]$. Ascertaining if these DAAs possess an inhibitory effect with regard to the occurrence and recurrence of HCC is important.

In conclusion, we analyzed the effect of IFN-based therapy on recurrence after curative treatment against HCC. This effect has been mainly analyzed in patients with surgical resection. Furthermore, there were only two studies investigating not only first recurrence but also second recurrence after curative treatment for initial HCC $[25,26]$. In the present study, SVR patients who had received curative treatment, including local ablation therapy and hepatic resection, had a favorable outcome compared with non-SVR patients. The similar trend was also observed in patients treated with RFA. These results appeared to be concordant with the findings of previous reports. Further prospective studies are necessary to confirm the effect of IFN in a larger number of patients with respect to cancer prevention.

\section{Abbreviations}

AASLD, American Association for the Study of Liver Diseases; AST, aspartate aminotransferase; ALT, alanine aminotransferase; $\mathrm{CT}$, computed tomography; DAA, direct-acting antiviral agent; ICG, indocyanine green; $\mathrm{HCC}$, hepatocellular carcinoma; $\mathrm{HCV}$, hepatitis $\mathrm{C}$ virus; IFN, interferon; NS, nonstructural protein; PEI, percutaneous ethanol injection; RFS, recurrence-free survival; SVR, sustained virological response; RFA, radiofrequency ablation.

\section{Acknowledgements}

The authors thank Dr. Fumihiko Kanai (Medical Corporation Eikenkai) for valuable discussions. 


\section{Conflicts of Interest}

Prof. Osamu Yokosuka received a research grant from Dainippon Sumitomo Pharma Co., Ltd., Chugai Pharmaceutical Co., Ltd., and MSD K.K. Dr. Tatsuo Kanda received lecture fees from Chugai Pharmaceutical Co., Ltd., and MSD K.K. All other authors have no conflict of interest to declare.

\section{References}

1. Ferlay J, Shin HR, Bray F, et al. Estimates of worldwide burden of cancer in 2008: GLOBOCAN 2008. Int J Cancer. 2010; 127: 2893-917.

2. Llovet JM, Burroughs A, Bruix J. Hepatocellular carcinoma. Lancet. 2003; 362: 1907-17.

3. Saito I, Miyamura T, Ohbayashi A, et al. Hepatitis C virus infection is associated with the development of hepatocellular carcinoma. Proc Natl Acad Sci U S A. 1990; 87: 6547-9.

4. Simonetti RG, Cammà $C$, Fiorello $F$, et al. Hepatitis $C$ virus infection as a risk factor for hepatocellular carcinoma in patients with cirrhosis. A case-control study. Ann Intern Med. 1992; 116: 97-102.

5. Chemello L, Cavalletto L, Bernardinello E, et al. The effect of interferon alfa and ribavirin combination therapy in naive patients with chronic hepatitis C. J Hepatol. 1995; 23(Suppl 2): 8-12.

6. Schvarcz $R$, Ando $Y$, Sönnerborg $A$, et al. Combination treatment with interferon alfa- $2 b$ and ribavirin for chronic hepatitis $C$ in patients who have failed to achieve sustained response to interferon alone: Swedish experience. J Hepatol. 1995; 23(Suppl 2): 17-21.

7. Ikeda K, Saitoh S, Arase Y, et al. Effect of interferon therapy on hepatocellular carcinogenesis in patients with chronic hepatitis type C: A long-term observation study of 1,643 patients using statistical bias correction with proportional hazard analysis. Hepatology. 1999; 29: 1124-30.

8. Sugiura N, Sakai Y, Ebara M, et al. Detection of hepatocellular carcinoma after interferon therapy for chronic hepatitis C: clinical study of 26 cases. J Gastroenterol Hepatol. 1996; 11: 535-9.

9. Ikeda K, Kobayashi M, Saitoh S, et al. Recurrence rate and prognosis of patients with hepatocellular carcinoma that developed after elimination of hepatitis $\mathrm{C}$ virus RNA by interferon therapy. A closed cohort study including matched control patients. Oncology. 2003; 65: 204-10.

10. Kubo S, Nishiguchi S, Hirohashi K, et al. Influence of previous interferon therapy on recurrence after resection of hepatitis $\mathrm{c}$ virus-related hepatocellular carcinoma. Jpn J Cancer Res. 2001; 92: 59-66.

11. Uenishi T, Kubo S, Hirohashi K, et al. Relationship between response to previous interferon therapy and postoperative recurrence of hepatitis $\mathrm{C}$ virus-related hepatocellular carcinoma. Hepatol Res. 2002; 24: 404-12.

12. McHutchison JG, Gordon SC, Schiff ER, et al. Interferon alfa-2b alone or in combination with ribavirin as initial treatment for chronic hepatitis C. Hepatitis Interventional Therapy Group. N Engl J Med. 1998; 339: 1485-92.

13. Heidelbaugh JJ, Bruderly M. Cirrhosis and chronic liver failure: part I. Diagnosis and evaluation. Am Fam Physician. 2006; 4: 756-62.

14. Bruix J, Sherman M. Practice Guidelines Committee, American Association for the Study of Liver Diseases. Management of hepatocellular carcinoma. Hepatology. 2005; 42: 1208-36.

15. Bruix J, Sherman M; American Association for the Study of Liver Diseases. Management of hepatocellular carcinoma: an update. Hepatology. 2011; 53: $1020-2$

16. Hoofnagle JH, Mullen KD, Jones DB, et al. Treatment of chronic non-A, non-B hepatitis with recombinant human alpha interferon. A preliminary report. $\mathrm{N}$ Engl J Med. 1986; 315: 1575-8.

17. Feld JJ, Hoofnagle JH. Mechanism of action of interferon and ribavirin in treatment of hepatitis C. Nature. 2005; 436: 967-72.

18. Kimura M, Haisa M, Uetsuka $H$, et al. TNF combined with IFN-alpha accelerates NF-kappaB-mediated apoptosis through enhancement of Fas expression in colon cancer cells. Cell Death Differ. 2003; 10: 718-28.

19. Herzer K, Hofmann TG, Teufel A, et al. IFN-alpha-induced apoptosis in hepatocellular carcinoma involves promyelocytic leukemia protein and TRAIL independently of p53. Cancer Res. 2009; 69: 855-62.

20. Shigeno $M$, Nakao $K$, Ichikawa $T$, et al. Interferon-alpha sensitizes human hepatoma cells to TRAIL-induced apoptosis through DR5 upregulation and NF-kappa B inactivation. Oncogene. 2003; 22: 1653-62.

21. Hasegawa K, Kokudo N, Makuuchi M, et al. Comparison of resection and ablation for hepatocellular carcinoma: a cohort study based on a Japanese nationwide survey. J Hepatol. 2013; 58: 724-9.

22. Feng K, Yan J, Li X, et al. A randomized controlled trial of radiofrequency ablation and surgical resection in the treatment of small hepatocellular carcinoma. J Hepatol. 2012; 57: 794-802.

23. Sugimachi K, Kinjo N, Ikebe M, et al. Significance of hepatic resection for hepatocellular carcinoma with sustained virological response to interferon therapy for chronic hepatitis C. Hepatol Res. 2013; 43: 605-9.
24. Sanefuji K, Kayashima H, Iguchi T, et al. Characterization of hepatocellular carcinoma developed after achieving sustained virological response to interferon therapy for hepatitis C. J Surg Oncol. 2009; 99: 32-7.

25. Miyatake H, Kobayashi $Y$, Iwasaki $Y$, et al. Effect of previous interferon treatment on outcome after curative treatment for hepatitis $C$ virus-related hepatocellular carcinoma. Dig Dis Sci. 2012; 57: 1092-101.

26. Sakae M, Kubo S, Takemura S, et al. Effect of interferon therapy on first and second recurrence after resection of hepatitis $C$ virus-related hepatocellular carcinoma. Hepatol Res. 2012; 43: 564-73.

27. Lok AS, Gardiner DF, Lawitz E, et al. Preliminary study of two antiviral agents for hepatitis C genotype 1. N Engl J Med. 2012; 366: 216-24.

28. Suzuki Y, Ikeda K, Suzuki F, et al. Dual oral therapy with daclatasvir and asunaprevir for patients with $\mathrm{HCV}$ genotype $1 \mathrm{~b}$ infection and limited treatment options. J Hepatol. 2013; 58: 655-62. 